\title{
Descrição da resposta ventilatória em provas de intensidades moderadas realizadas em cicloergômetro
}

\author{
Artigo Original
}

\section{Samuel Sarmiento}

samu_sarmiento@yahoo.es

Juan Manuel García-Manso'

igarcia@def.ulpgc.es

Juan Manuel Martín-González' igonzalez@dfis.ulpgc.es

Javier Calderón ${ }^{2}$

i.calderon@yahoo.com

Pedro Benito ${ }^{2}$

bebito_benito@yahoo.com
'Departamento de Educação Física - Universidad de Las Palmas de Gran Canaria Espanha

${ }^{2}$ Faculdade de Ciências da Atividade Física e Esporte - Universidad Politécnica de Madrid - Espanha

Sarmiento S, García-Manso JM, Martín-González JM, Calderón J, Benito P. Descrição da resposta ventilatória em provas de intensidades moderadas realizadas em cicloergômetro. Fit Perf J. 2008 mar-abr;7(2):99-106.

RESUMO: Introdução: $O$ trabalho analisa e descreve o comportamento do sistema respiratório e o sistema de contribuição de oxigênio durante uma prova de intensidade moderada realizada em um cicloergômetro. Materiais e Métodos: Utilizou-se uma

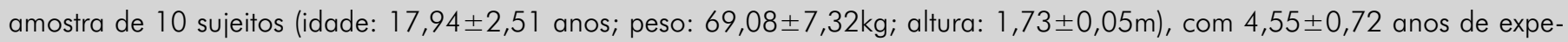
riência em ciclismo. Todos fizeram duas provas. A primeira, uma prova incremental até o esgotamento ( $\Delta$ de $5 \mathrm{~W}$ cada $12 \mathrm{~s}$, a partir de $100 \mathrm{~W}$ ) que serviu para determinar $\circ \mathrm{VO}_{2 \text { máx }}$ os limiares ventilatórios e a carga de trabalho para a prova de carga estável em intensidade moderada (PEM). Na PEM trabalharam durante 30s com uma carga individualmente determinada $(249,50 \pm 18,02 \mathrm{~W}$ a 3,64 $\pm 0,38 \mathrm{~W} \cdot \mathrm{kg}^{-1}$ ). Resultados: A carga na PEM foi de 58,82 $\pm 2,48 \%$ da carga máxima atingida na prova incremental e $67,60 \pm 5,46 \%$ da carga no limiar anaeróbico na mesma prova. Discussão: Em nosso estudo, o $\mathrm{VO}_{2}$ aumentou à razão de

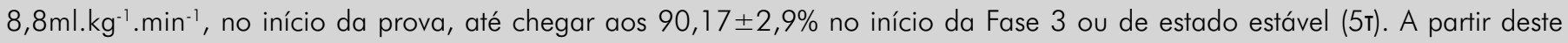
ponto, os sujeitos estudados mostraram um aumento suave e progressivo do $\mathrm{VO}_{2}$, até aproximadamente os $12 \mathrm{~min}$ de exercício, quando estabilizaram a resposta até o final da prova.

Palavras-chave: Exercício, Consumo de Oxigênio, Limiar Anaeróbio.

Endereço para correspondência: Facultad de Ciencias de la Actividad Física y el Deporte - Campus Universitario de Tafira 25017 Las Palmas de Gran Canaria - Espanha 
Description of the ventilatory response in moderate intensity tests accomplished in cycloergometer

Introduction: This work analyzes and describes the behavior of the respiratory system and the system of oxygen contribution during a moderate intensity test accomplished in a cycloergometer. Materials and Methods: A sample composed of 10 individuals (age: $17.94 \pm 2.51$ years old; weight: $69.08 \pm 7.32 \mathrm{~kg}$; height: $1.73 \pm 0.05 \mathrm{~m}$ ), with $4.55 \pm 0.72$ years of experience in cycling. All of them were submitted to two tests. The first one, an incremental test until exhaustion ( $\Delta$ of $5 \mathrm{~W}$ each $12 \mathrm{~s}$, starting from $100 \mathrm{~W}$ ), which served to determine the $\mathrm{VO}_{2 \text { máx }}$ the ventilatory threshold and the workload for the test of stable load in moderate intensity (TSM). In the TSM they worked during 30s with an individually determined load $\left(249.50 \pm 18.02 \mathrm{~W}\right.$ a $\left.3.64 \pm 0.38 \mathrm{~W} \cdot \mathrm{kg}^{-1}\right)$. Results: The load in the TSM was $58.82 \pm 2.48 \%$ of the maximum load reached in the incremental test and $67.60 \pm 5.46 \%$ of the load reached in the anaerobic threshold in the same test. Discussion: In our study, the $\mathrm{VO}_{2}$ increased in $8.8 \mathrm{ml} . \mathrm{kg}^{-1} . \mathrm{min}^{-1}$ in the beginning of the test, until reaching $90.17 \pm 2.9 \%$ in the beginning of phase 3 or stable state (5T). From this point on, the studied individuals showed a smooth and progressive increase of $\mathrm{VO}_{2}$, until, approximately, $12 \mathrm{~min}$ of exercise, when they stabilized the response until the end of the test.

Keywords: Exercise, Oxygen Consumption, Anaerobic Threshold.
Descripción de la respuesta ventilatoria en pruebas de moderada intensidad realizadas en cicloergómetro

Introducción: El trabajo analiza y describe el comportamiento del sistema respiratorio y el sistema de aporte de oxígeno durante una prueba de moderada intensidad realizada en un cicloergómetro. Materiales y Métodos: Se utilizó una muestra de 10 sujetos (edad: 17,94 $\pm 2,51$ años; peso: 69,08 $\pm 7,32 \mathrm{~kg}$;

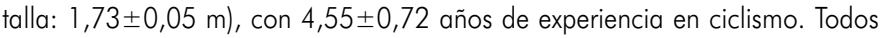
hicieron dos pruebas. La primera una prueba incremental hasta el agotamiento ( $\Delta$ de $5 \mathrm{~W}$ cada $12 \mathrm{~s}$, a partir de $100 \mathrm{~W}$ ) que sirvió para determinar el $\mathrm{VO}_{2 \text { máx }}$ los limiares ventilatorios y la carga de trabajo para la prueba de carga estable a moderada intensidad (PEM). En la PEM trabajaron durante 30 min con una carga individualmente determinada $\left(249,50 \pm 18,02 \mathrm{~W}\right.$ ó $\left.3,64 \pm 0,38 \mathrm{~W} \cdot \mathrm{kg}^{-1}\right)$. Resultados: La carga en la PEM fue del $58,82 \pm 2,48 \%$ de la carga máxima alcanzada en la prueba incremental y un 67,60 5,46\% de la carga al limiar anaeróbico en la misma prueba. Discusión: En nuestro estudio, el $\mathrm{VO}_{2}$ aumentó a razón de $8,8 \mathrm{ml} \cdot \mathrm{kg}^{-1} \cdot \mathrm{min}^{-1}$ al inicio de la prueba hasta llegar al 90,17 $\pm 2,9 \%$ al inicio de la fase III o de estado estable (5T). A partir de este punto, los sujetos estudiados mostraron un incremento suave y progresivo del $\mathrm{VO}_{2}$ hasta aproximadamente los 12 min de ejercicio, momento en que estabilizaros la respuesta hasta el final de la prueba.

Palabras clave: Ejercicio, Consumo de Oxígeno, Umbral Anaerobio.

\section{INTRODUÇÃO}

As cargas de intensidade moderada são cargas de média e longa duração que se utiliza para o desenvolvimento da capacidade aeróbica do esportista. Estas cargas são empregadas, geralmente, com intensidades ao redor de $60-75 \%$ do consumo máximo de oxigênio $\left(\mathrm{VO}_{2 \text { móx }}\right)$, o que corresponde aos valores próximos de $65-75 \%$ da velocidade aeróbica máxima (VAM) ou a $70-80 \%$ do limiar anaeróbico. Isso supõe trabalhar com freqüência cardíaca (FC) de aproximadamente 150 a 170 batimentos por minutos e concentrações de lactato sanguíneo que oscilam entre $3 \mathrm{mmol} . \mathrm{L}^{-1}$ e $6 \mathrm{mmol} . \mathrm{L}^{-1}$. Nelas, desde os primeiros instantes de atividade, desencadeia-se uma resposta intensa do sistema respiratório com a intenção de fornecer a quantidade suficiente de $\mathrm{O}_{2}$ que o organismo necessita. $\mathrm{O}$ comportamento dos parâmetros ventilatórios apresenta uma cinética individual, que depende da intensidade e características da carga de trabalho utilizada e vem condicionada, entre outros fatores, pelo nível de treinamento que possua o sujeito' ${ }^{1}$ o o nível de ativação prévia dos mesmos ${ }^{2,3,4}$.

Para isso, o sistema respiratório responde aumentando o número de respirações $\left(F_{R}\right)$ e a profundidade de cada uma delas $\left(V_{C}\right)$. Ambos os parâmetros condicionam a quantidade de ar que o sujeito movimenta por unidade de tempo $\left(V_{E}\right)$, facilitando os mecanismos de transporte de oxigênio para o interior do organismo. No entanto, a forma como evoluem estas variáveis não é exatamente igual quando o sujeito realiza um exercício.

O controle destes processos não é totalmente conhecido, mesmo que na atualidade tenham sido identificados diversos mecanismos que são regulados a partir do sistema nervoso central (SNC). Os centros respiratórios localizados no SNC (bulbo raquidiano e protuberância) são os encarregados de regular o ritmo respiratório em função da informação que recebam dos diversos pontos do organismo (quimiorreceptores, mecanorreceptores, etc. $)^{\mathbf{5 , 6}}$. No início do século XX, Krogh \& Lindhard ${ }^{7}$ demonstraram que, com o exercício, se produzia um abrupto aumento da resposta ventilatória desde os primeiros segundos de esforço. Inclusive, desde uns segundos antes da atividade, o organismo se prepara para o exercício e responde aumentando a resposta ventilatória. Este comportamento demonstra que a resposta cardio-ventilatória tem uma origem cortical.

O objetivo de nosso trabalho é fazer uma descrição pormenorizada da resposta ventilatória e da resposta integrada de contribuição de oxigênio $\left(\mathrm{VO}_{2}\right)$ como respostas prolongadas e de moderada intensidade.

\section{MATERIAIS E MÉTODOS}

\section{Aprovação do esłudo}

Todos os sujeitos assinaram um termo de consentimento livre e esclarecido, de acordo com as Diretrizes Éticas da Declaração de Helsinque, que foi aceito pelo Comitê de Ética da Universidad de Las Palmas de Gran Canaria. 


\section{Amostra}

Foi utilizada uma amostra de 10 sujeitos jovens (idade: $17,94 \pm 2,51$ anos; peso: $69,08 \pm 7,32 \mathrm{~kg}$; altura: 1,73 $\pm 0,05 \mathrm{~m})$, com 4,55 $\pm 0,72$ anos de experiência em competições de ciclismo em estrada e mountain bike. Os volumes de treinamento anuais destes ciclistas são, aproximadamente, $15.477 \pm 6.003 \mathrm{~km}$ anuais (categoria: $28.800-6.720 \mathrm{~km}$ anuais) entre os ciclistas de estrada e aproximadamente 7.920 $\pm 20 \mathrm{~km}$ anuais (categoria: 8.640-7$.200 \mathrm{~km}$ anuais) entre os ciclistas de mountain bike. Todos tinham experiência prévia em provas de esforço sobre cicloergômetro.

Os sujeitos foram informados dos riscos e características do estudo. Todos assinaram um documento de aceitação, que respondia às diretrizes éticas estabelecidas na declaração de Helsinki pela Associação Médica Mundial para a investigação com seres humanos.

Na Tabela 1 estão mostradas as características antropométricas (altura, peso corporal, IMC e porcentagem de gordura) expressas em valores médios \pm desvios padrões da amostra utilizada nas provas de carga estável em intensidade moderada (PEM).

A Tabela 2 mostra as características fisiológicas (resposta cardíaca máxima e de repouso e $\mathrm{VO}_{2 \text { máx }}$ ) e de carga (carga absoluta e relativa) com a qual trabalharam estes sujeitos nas PEM.

\section{Características da prova}

Todos os sujeitos fizeram duas provas no cicloergômetro. A primeira foi uma prova incremental até o esgotamento (incrementos de $5 \mathrm{~W}$ a cada 12s, a partir de uma carga inicial de 100W) que serviu para a determinação do $\mathrm{VO}_{2 \text { máx }}$ os limiares ventilatórios (VT1 e VT2) e a carga à que deviam fazer a PEM.

Na prova estável, os sujeitos trabalharam durante $30 \mathrm{~min}$ com uma carga individualmente determinada $(249,50 \pm 18,02 \mathrm{~W}$ ou $\left.3,64 \pm 0,38 \mathrm{~W} \cdot \mathrm{kg}^{-1}\right)$. Esta carga supõe uma intensidade média de $58,82 \pm 2,48 \%$ da carga máxima atingida na prova incremental e 67,60 $5,46 \%$ da carga de trabalho com a qual se alcançou - limiar anaeróbico na mesma prova. A cadência de pedalada exigida durante a prova foi estabelecida em 80-90 rotações por minuto. Na Tabela 2 apresentam-se os valores médios de carga de trabalho (W) e resposta funcional teórica $\left(\mathrm{VO}_{2}\right.$ e $\left.\mathrm{FC}\right)$, expressos em valores relativos (\%) aos que se programou a PEM. Estes valores foram determinados a partir dos valores máximos alcançados na prova incremental (PI), e aos valores que cada sujeito

Tabela 1 - Características antropométricas da amostra (altura, peso corporal, IMC e porcentagem de gordura) expressas em valores médios \pm desvios padrões

\begin{tabular}{lc}
\hline idade (anos) & $17,94 \pm 2,51$ \\
altura $(\mathrm{m})$ & $1,73 \pm 0,05$ \\
peso corporal $(\mathrm{kg})$ & $69,08 \pm 7,32$ \\
Índice de Massa Corporal $\left(\mathrm{kg} \cdot \mathrm{m}^{-2}\right)$ & $22,94 \pm 1,30$ \\
porcentagem de gordura $(\%)$ & $8,91 \pm 1,45$ \\
superfície corporal $\left(\mathrm{m}^{2}\right)$ & $1,82 \pm 0,12$ \\
área frontal $\left(\mathrm{m}^{2}\right)$ & $0,32 \pm 0,02$ \\
\hline
\end{tabular}

mostrava quando se alcançava, na mesma prova, a intensidade correspondente ao VT2.

\section{Material}

A freqüência cardíaca se registrou com um freqüencímetro cardíaco Polar S810i (Polar Electro Oy, Finland). O registro da freqüência cardíaca através do freqüencímetro cardíaco é um método recentemente validado, mediante ECG, por vários estudos ${ }^{\mathbf{8}, 9}$.

Os parâmetros ventilatórios foram analisados com um equipamento Jaeger Oxicon Pró ${ }^{\circledR}$ (Viasys Healthcare, Alemanha). $\bigcirc$ analisador estava conectado a um computador de mesa com o Software LabManager v4.52.i., que permite a análise e representação gráfica dos dados. $\bigcirc$ equipamento foi calibrado antes da realização de cada uma das provas, mediante um sistema de autocalibração que despreza as variações de $\pm 2 \%$ com relação aos valores padronizados. Este sistema computarizado de análise dos gases foi validado durante a aplicação de diversas intensidades de exercício ${ }^{10}$.

As provas foram realizadas sobre um cicloergômetro Jaerger ER800 (Viasys Healthcare, Alemanha), de faixa de 25Watts a 1000 Watts, com incrementos mínimos de 1 Watt.s $^{-1}$ e dispositivo de freio eletromecânico, no qual os sujeitos mantiveram uma posição convencional. $\bigcirc$ selim e o guidom foram ajustados para cada sujeito em função de sua altura, buscando a maior comodidade possível. Para as medições de prova dos ciclistas foram usados pedais automáticos, já que o sujeito realizava a prova com seu próprio calçado habitual de ciclismo.

\section{Tratamento dos sinais}

Os parâmetros ventilatórios eram exportados do software do analisador e analisados no programa $M A T L A B^{\circledR}$ para realizar as representações gráficas e determinar os valores utilizados no estudo.

O estudo da FC se fez a partir da variabilidade temporária existente entre cada batida (VFC) que, posteriormente, foi analisada mediante um protocolo no domínio tempo-freqüência (análise Wavelet). A análise Wavelet tem a vantagem de analisar o sinal no domínio tempo-escala, usando algoritmos de cálculos rápidos e fáceis de implementar. Isto permite fazer uma "radiografia" muito precisa da evolução das freqüências e, além disso, permite analisar diretamente sinais de compor-

Tabela 2 - Características fisiológicas (resposta cardíaca máxima e de repouso e consumo máximo de oxigênio) e de cargas (cargas absoluta e relativa) da PEM

\begin{tabular}{lc}
\hline carga de trabalho absoluta $(\mathrm{W})$ & $249,50 \pm 18,02$ \\
carga de trabalho relativa $\left(\mathrm{W} \cdot \mathrm{kg}^{-1}\right)$ & $3,64 \pm 0,38$ \\
carga de trabalho relativa $\left(\%\right.$ vs. $\left.\mathrm{W}_{\text {máx }}\right)$ & $58,82 \pm 2,48$ \\
carga de trabalho relativa $\left(\%\right.$ vs. $\left.\mathrm{VO}_{2 \text { máx }}\right)$ & $65,28 \pm 3,26$ \\
$\mathrm{FC}_{\text {reposo }}$ (batidas/min) & $52,50 \pm 8,72$ \\
$\mathrm{FC}_{\text {máx }}$ (batidas $\left./ \mathrm{min}\right)$ & $177,78 \pm 5,97$ \\
$\mathrm{VO}_{2 \text { máx }}\left(\right.$ L. $\left.\mathrm{min}^{-1}\right)$ & $3,85 \pm 0,26$ \\
\hline
\end{tabular}


tamento não-estacionário, já que os componentes espectrais do sinal objeto de estudo variam com o transcurso do tempo (sinal cardíaco em fase de esforço). Estas metodologias proporcionam ao observador a possibilidade de detectar mudanças de comportamento no domínio tempo e no domínio freqüência de forma simultânea.

\section{Análise estatística dos dados}

A análise estatística se fez com o pacote SPSS para Windows versão 13.0. Na descrição da amostra e na descrição da cinética dos parâmetros funcionais, medidos em cada uma das provas (crescente e estável), foi realizada uma estatística descritiva básica nas quais, em função das necessidades, se calculou média, mediana, desvio padrão, máximo e mínimo do valor correspondente.

Quando se fez necessário quantificar e valorar mudanças na cinética dos parâmetros funcionais (cardíacos e respiratórios), realizou-se uma estatística comparativa, empregando-se o cálculo de comparação de médias relacionadas (Wilcoxon). ○ programa calculava, para cada variável, a média, o desvio padrão e o erro típico da média. Para cada par de variáveis, determina a correlação, a diferença média entre as médias, a prova T para o intervalo de confiança da diferença das médias e a prova T para o intervalo da diferença nas médias. Além disso, proporciona desvio padrão e erro típico da diferença entre as médias. As decisões estatísticas foram calculadas tomando

Tabela 3 - Valores médios da cinética do VE, VC, FR e VO ao começo e ao final (5T) da fase exponencial rápida da PEM

\begin{tabular}{|c|c|c|c|}
\hline fase variáveis & início prova & 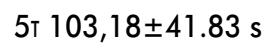 & final prova \\
\hline VE $\left(L \cdot \min ^{-1}\right)$ & $51,05 \pm 5,80$ & $77,24 \pm 6,20$ & $98,91 \pm 10,41$ \\
\hline VC (L) & $1,92 \pm 0,10$ & $2,42 \pm 0,20$ & $2,42 \pm 0,20$ \\
\hline $\mathrm{FR}$ (resp/min) & $27,57 \pm 4,30$ & $32,65 \pm 3,30$ & $45,76 \pm 5,80$ \\
\hline
\end{tabular}

Tabela 4 - Evolução da média dos valores correspondentes aos parâmetros ventilatórios estudados (VE, VC e FR) em cada trecho da segunda parte de Uma PEM

\begin{tabular}{lccccccc}
\hline Parâmetro & Trecho 1 & Trecho 2 & Trecho 3 & Trecho 4 & Trecho 5 & Trecho 6 & Trecho 7 \\
\hline VE (L.min $\left.{ }^{-1}\right)$ & $82,20 \pm 6,26$ & $87,90 \pm 7,29$ & $91,00 \pm 8,15$ & $92,42 \pm 8,61$ & $94,77 \pm 9,03$ & $96,68 \pm 9,68$ & $98,91 \pm 10,48$ \\
VC(L) & $2,36 \pm 0,26$ & $2,32 \pm 0,27$ & $2,27 \pm 0,24$ & $2,25 \pm 0,24$ & $2,24 \pm 0,23$ & $2,20 \pm 0,24$ & $2,36 \pm 0,24$ \\
FR (Resp/min) & $34,78 \pm 4,21$ & $38,18 \pm 4,99$ & $40,11 \pm 5,83$ & $41,49 \pm 5,55$ & $43,04 \pm 5,93$ & $43,90 \pm 5,42$ & $45,76 \pm 5,88$ \\
\hline
\end{tabular}

Tabela 5 - Os valores de 1 т a 5 т (em segundos) com seus equivalentes em $\mathrm{VO}_{2}$ (L.min-1) e \% do VO $\mathrm{V}_{2}$ em relação ao máximo alcançado por este parâmetro durante a PEM

\begin{tabular}{|c|c|c|c|c|c|c|}
\hline fases & início & $1 \mathrm{~T}$ & $2 \mathrm{~T}$ & Зт & $4 \mathrm{~T}$ & 5т \\
\hline variáveis & prova & $20,64 \pm 8.37$ & $41,27 \pm 16.73$ & $61,91 \pm 25.10$ & $83,54 \pm 33.46$ & $103,18 \pm 41.83$ \\
\hline $\mathrm{VO}_{2}\left(\right.$ L. $\left.\mathrm{min}^{-1}\right)$ & $2,44 \pm 0,3$ & $2,81 \pm 0,2$ & $3,19 \pm 0,2$ & $3,40 \pm 0,2$ & $3,43 \pm 0,2$ & $3,47 \pm 0,2$ \\
\hline$\% \mathrm{VO}_{2 \text { máx }}$ alcançado na prova & $63,47 \pm 8,1$ & $73,01 \pm 4,7$ & $82,79 \pm 3,9$ & $88,37 \pm 4,7$ & $89,25 \pm 3,1$ & $90,17 \pm 2,9$ \\
\hline
\end{tabular}

Tabela 6 - Evolução do VO (L.min $^{-1}$ ) ao longo dos 28 mitos finais da PEM

\begin{tabular}{lccccccc}
\hline & $\begin{array}{c}\mathrm{VO}_{2} \\
\mathrm{Trecho} \mathrm{1}\end{array}$ & $\begin{array}{c}\mathrm{VO}_{2} \\
\mathrm{Trecho} \mathrm{2}\end{array}$ & $\begin{array}{c}\mathrm{VO}_{2} \\
\mathrm{Trecho} \mathrm{3}\end{array}$ & $\begin{array}{c}\mathrm{VO}_{2} \\
\text { Trecho 4 }\end{array}$ & $\begin{array}{c}\mathrm{VO}_{2} \\
\text { Trecho 5 }\end{array}$ & $\begin{array}{c}\mathrm{VO}_{2} \\
\text { Trecho 6 }\end{array}$ & $\begin{array}{c}\mathrm{VO}_{2} \\
\text { Trecho 7 }\end{array}$ \\
\hline $\mathrm{VO}_{2}\left(\mathrm{~L} \cdot \mathrm{min}^{-1}\right)$ & $3,62 \pm 0,23$ & $3,68 \pm 0,23$ & $3,73 \pm 0,24$ & $3,73 \pm 0,24$ & $3,76 \pm 0,26$ & $3,77 \pm 0,26$ & $3,75 \pm 0,31$ \\
$\mathrm{VO}_{2}(\%)$ & $94,08 \pm 1,85$ & $95,71 \pm 2,26$ & $96,90 \pm 2,09$ & $96,92 \pm 1,21$ & $97,64 \pm 0,80$ & $98,02 \pm 1,26$ & $97,26 \pm 3,29$ \\
$\Delta \mathrm{VO}_{2}\left(\mathrm{~L} \cdot \mathrm{min}^{-1}\right)$ & $0,08 \pm 0,06$ & $0,14 \pm 0,08$ & $0,19 \pm 0,08$ & $0,19 \pm 0,08$ & $0,22 \pm 0,07$ & $0,23 \pm 0,06$ & $0,21 \pm 0,13$ \\
\hline
\end{tabular}

Tabela 7 - Incrementos do $\mathrm{VO}_{2}$ em cada trecho da PEM

\begin{tabular}{|c|c|c|c|c|c|}
\hline \multirow{2}{*}{ Variáveis } & \multicolumn{5}{|c|}{ Diferenças relacionadas } \\
\hline & média & desvio padrão & erro médio & $t$ & significação bilateral \\
\hline diferenças $\mathrm{VO}_{2}$ (Trechos 1 a 2 ) & $+0,06$ & 0,03 & 0,01 & $-6,30$ & 0,000 \\
\hline diferenças $\mathrm{VO}_{2}$ (Trechos 2 a 3) & $+0,05$ & 0,03 & 0,01 & $-4,19$ & 0,002 \\
\hline diferenças $\mathrm{VO}_{2}$ (Trechos 3 a 4 ) & 0,00 & 0,05 & 0,02 & 0,00 & 1,000 \\
\hline diferenças $\mathrm{VO}_{2}$ (Trechos 4 a 5) & $+0,03$ & 0,05 & 0,02 & $-1,92$ & 0,087 \\
\hline diferenças $\mathrm{VO}_{2}$ (Trechos 5 a 6 ) & $+0,01$ & 0,03 & 0,01 & $-1,42$ & 0,189 \\
\hline diferenças $\mathrm{VO}_{2}$ (Trechos 6 a 7) & $-0,03$ & 0,09 & 0,03 & 0,90 & 0,389 \\
\hline
\end{tabular}


um nível de significação de $p<0,05$. A cada série de dados, antes de realizar a comparação de médias, aplicou-se provas de normalidade e de homogeneidade de variâncias que assegurassem uma distribuição normal dos dados das séries (prova de Kolmogorov-Smirnof).

A representação gráfica dos valores se fez automaticamente, com as possibilidades que proporcionava o pacote utilizado. Normalmente se utilizaram os valores brutos das séries dos sinais utilizadas, mesmo que, em algumas ocasiões, determinou-se sua tendência mediante o cálculo de transformadas wavelet discretas, que eram inseridas sobre o sinal. Uma das representações gráficas utilizadas neste trabalho foi o diagrama de caixas (box-plot).

\section{Dados}

Na Tabela 3 são mostrados os valores médios da cinética do $V_{E^{\prime}} V_{C^{\prime}} F_{R}$ e $V O_{2}$ ao começo e no final (5T) da fase exponencial rápida da prova estável moderada.

Na Tabela 4 mostra-se a evolução da média dos valores correspondentes aos parâmetros ventilatórios estudados $\left(V_{E^{\prime}}, V_{C}\right.$ e $\left.F_{R}\right)$, em cada trecho da segunda parte de uma prova prolongada de intensidade moderada.

Figura 1 - Exemplo de como evoluem os parâmetros ventilatórios durante uma PEM de um dos sujeitos da amostra

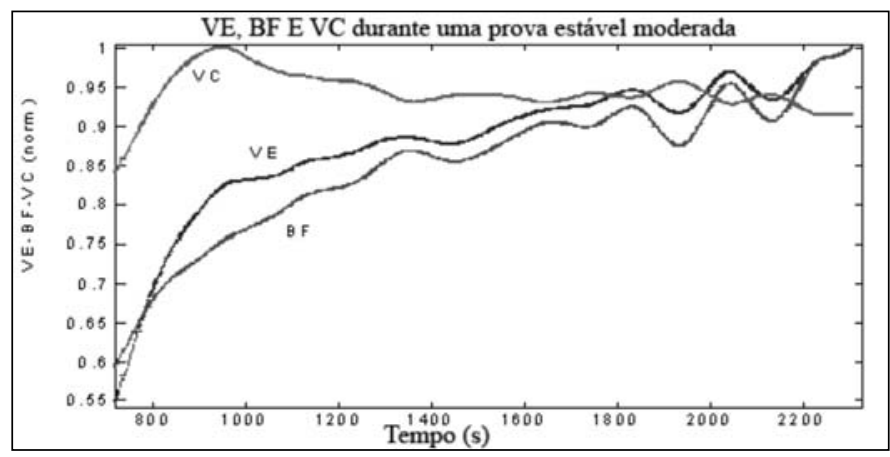

Figura 2 - Exemplo de como evolui o $\mathrm{VO}_{2}\left(\right.$ L.min $\left.{ }^{-1}\right)$ em um dos sujeitos da amostra

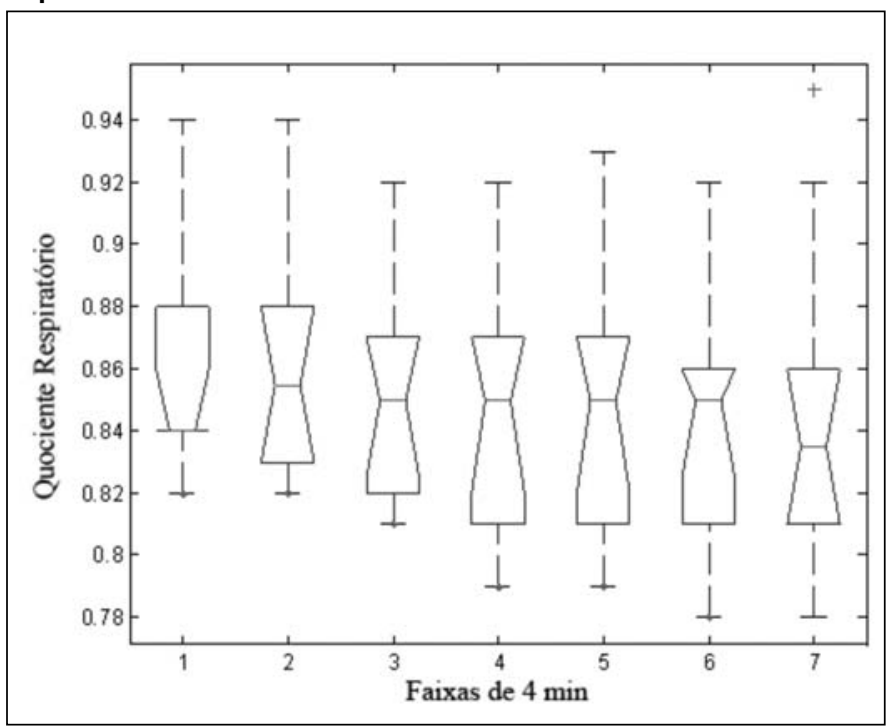

Na Tabela 5 aparecem os valores de 1т a 5т (em segundos) com seus equivalentes em $\mathrm{VO}_{2}\left(\mathrm{~L}_{\mathrm{m}} \mathrm{min}^{-1}\right)$ e \% do $\mathrm{VO}_{2}$, em relação ao máximo alcançado por este parâmetro durante a prova.

$\mathrm{Na}$ Tabela 6 se apresenta a evolução do $\mathrm{VO}_{2}$ ao longo dos $28 \mathrm{~min}$ finais de prova. $\mathrm{O} \mathrm{VO}_{2}$ é expresso em valores absolutos (L. $\left.\mathrm{min}^{-1}\right)$ e relativos (\%), assim como os incrementos médios do $\mathrm{VO}_{2} \mathrm{em}$ relação aos valores máximos. Os resultados são mostrados em valores médios, correspondentes a cortes de $4 \mathrm{~min}$ de prova, a partir dos 120s do início.

$\mathrm{Na}$ Tabela 7 se mostram os incrementos de $\mathrm{VO}_{2}$ em cada trecho da prova, assim como os resultados das comparações de médias correspondentes a este parâmetro.

Na Figura 1 há um exemplo de como evolvem os parâmetros ventilatórios, com dados pertencentes a um sujeito da mostra. Os valores foram normalizados em relação ao valor máximo alcançado na prova. A linha central corresponde ao comportamento da $V_{E}$, enquanto na linha superior e na linha inferior se representam as evoluções do $V_{C}$ e de $F_{R^{\prime}}$ respectivamente.

A Figura 2 apresenta um exemplo de como evolui o $\mathrm{VO}_{2}$ em um dos sujeitos da amostra. Os valores são expressos, respiração a respiração, em L. $\mathrm{min}^{-1}$. Também é mostrado o valor que corresponderia à fase de estado estável.

A Figura 3 mostra, em um box-plot, a evolução do quociente respiratório (RER) durante a segunda fase da prova mantida de moderada intensidade.

\section{Discussão}

Neste parágrafo analisaremos a prova estável por dois ângulos: o estudo da cinética ventilatória e o estudo da cinética do $\mathrm{VO}_{2}$.

\section{Respesta ventilatória $\left(V_{E}, V_{C} \in F_{R}\right)$} Figura 3 - Box-Plot que mostra a evolução do RER durante a segunda fase da PEM

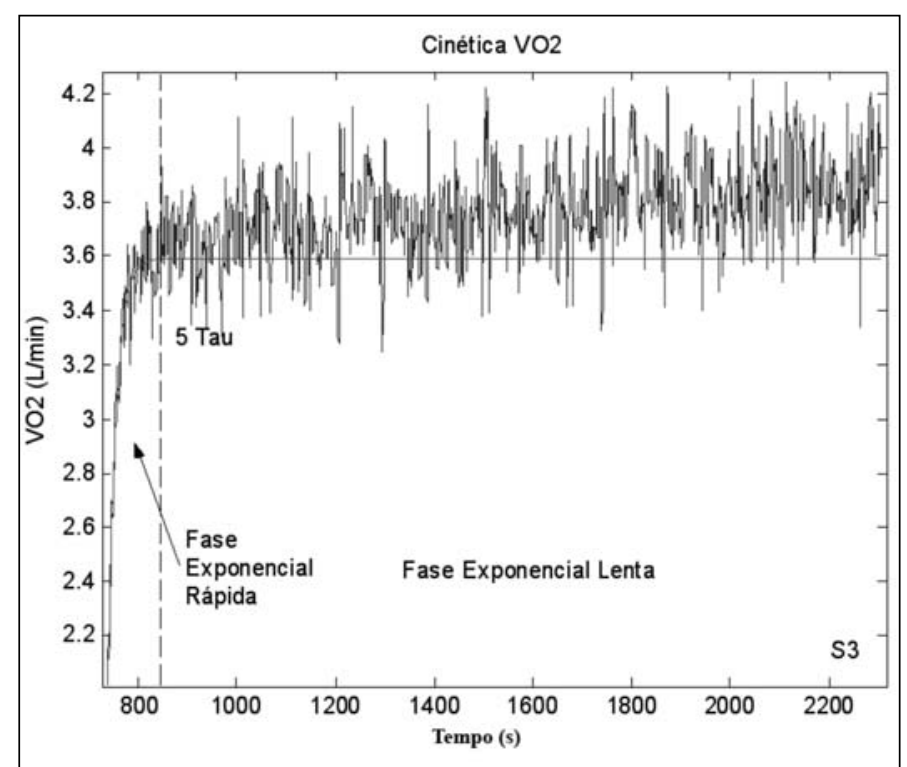


Em nossa amostra, a ventilação aumenta desde o início da prova. Este incremento de $V_{E}$ se produz de forma rápida nos primeiros minutos de prova (Tabela 3), e de maneira suave até o final da mesma (Tabela 4). Os valores médios da $V_{E}$ ao final da primeira fase (5 $\mathrm{T}$ do $\mathrm{VO}_{2}$ ) só chegam a valores de 76,03\% $\left(77,24 \pm 6,20 \mathrm{~L}\right.$. $\mathrm{min}^{-1}$ vs. 98,91 $\left.\pm 10,41 \mathrm{~L} \cdot \mathrm{min}^{-1}\right)$ dos máximos atingidos durante a prova (Tabela 2). Estes valores parecem coincidir com os do final da fase de incremento rápido deste parâmetro $\left(V_{E}\right)$.

O primeiro parâmetro ventilatório que alcança seus valores máximos é o $V_{C}$ (Figura 1), dando-se esta situação pouco depois de terminar a primeira fase ou no final da mesma (fase rápida; $5 \mathrm{~T}$ do $\mathrm{VO}_{2}$ ), para continuar com uma diminuição progressiva e moderada do valor até o final do exercício (Tabela 4). Este aumento é praticamente linear até o valor de $3 \mathrm{~T}$, o qual chega até quase $75 \%$ do valor máximo, para reduzir esse ritmo de aumento até o final da fase. Esta resposta vem condicionada pelos níveis de $\mathrm{CO}_{2}$ $\left(\mathrm{VCO}_{2}\right)$ e, em conseqüência, pela intensidade de trabalho.

Diferente do que ocorre com $\circ V_{C^{\prime}}$ o ritmo respiratório $\left(F_{R}\right)$, durante a execução de esforços moderados, adapta-se progressivamente à demanda de $\mathrm{O}_{2}$, aumentando paulatinamente seus valores até o final da prova. Não obstante, sua dinâmica também mostra um comportamento em duas fases. Durante a primeira fase é gerada uma resposta rápida e intensa que apresenta um aumento, mais ou menos linear, até em final da mesma. No final da fase, o valor médio de $F_{R}$ de nosso estudo apresenta valores de $32,7 \pm 3,3$ respirações. $\mathrm{min}^{-1}$, ainda longe das 45 respirações. $\mathrm{min}^{-1}$ que quase todos nossos sujeitos alcançaram de valor máximo durante o esforço.

Depois da rápida resposta inicial de todos os parâmetros, só $V_{E}$ e $F_{R}$ continuam se incrementando durante a segunda parte da prova. Não obstante, nenhum dos dois parâmetros apresenta um aumento intenso, mas evoluem de forma moderada até o final do esforço. Pelo contrário, $O V_{C}$ tende a estabilizar-se, ou inclusive a diminuir, com estas intensidades de trabalho, sempre que a fadiga acumulada não altere a resposta.

Importante é a vinculação existente entre $\circ V_{C}$ e o tempo inspiratório $\left(T_{i}\right)$. Pensemos que, inicialmente, $V_{C}$ se modifica em função da demanda metabólica, que provoca o esforço a partir da informação que o SNC recebe de quimiorreceptores periféricos, a qual, posteriormente, adaptará $V_{C}$ à demanda e regulará a $F_{R}$ a partir dos tempos de inspiração e expiração. Quando a intensidade é baixa (<50\%), a $V_{E}$ aumenta fundamentalmente a partir de $V_{C}$. Este parâmetro aumenta inicialmente até alcançar valores próximos a $1000 \mathrm{ml}$, de forma paralela à $F_{R^{\prime}}$ sem que por isso o faça $T_{i}$. A seguir, aumenta a $V_{E}$ com a carga a partir de um aumento de $V_{C^{\prime}}$ acompanhado tudo isto de uma queda de $T_{i}$. Posteriormente, o $V_{C}$ se estabiliza e a $F_{R}$ aumenta de forma desproporcionada quando a carga é incremental, provocando a perda de linearidade da $V_{E}$ com relação à carga. Este aumento da $V_{E}$ não se observou em nosso trabalho.
Durante o transcurso da prova, os valores da média de $V_{E}$ aumentam lentamente, mesmo que de forma significativa, até o final $\left(\Delta V_{E}: 20,33 \% ; p<0,01\right)$. Estes incrementos de $V_{E}$ são gerados a partir dos mencionados aumentos da $F_{R}(\Delta F R: 31,57 \% ; p<0,01)$. Paralelo a esse aumento de $F_{R^{\prime}}$ se observa uma redução progressiva do número de batidas por respiração (T1: 4,51 batidas/ respiração vs. T7: 3,80 batidas/respiração; $p>0,01)$.

Desde os primeiros instantes da atividade, o consumo de energia aumenta rapidamente em relação aos níveis de repouso. Esta quantidade é sempre proporcional à intensidade de trabalho e, em conseqüência, desencadeia uma resposta cardiorrespiratória conforme as necessidades do organismo e das características das cargas a que é submetido. Tal inércia metabólica inicial apresenta uma resposta muito rápida que, inclusive, se antecipa ao comportamento retardado que mostra a extração de $\mathrm{O}_{2}$ e a desnaturação da hemoglobina. Hogan ${ }^{11}$ e DeLorey et al. ${ }^{\mathbf{1 2}}$, cifram o atraso deste comportamento em $\approx 13 \mathrm{~s}$. Em nosso trabalho não se observa este comportamento, visto que os sujeitos iniciam a atividade imediatamente após a realização do aquecimento, não havendo intervalo entre eles.

\section{Resposta do $\mathbf{V} \boldsymbol{\bullet}_{2}$}

$\bigcirc \mathrm{VO}_{2}$ proporciona informação da atividade integrada dos sistemas respiratório (SAO), cardiovascular e neuromuscular. Tal resposta está condicionada, entre outros fatores, pela intensidade da carga e pela demanda energética da musculatura ativa, que será rápida e intensa desde os primeiros instantes do início do exercício.

Sugeriu-se que na fase inicial de um exercício o aumento rápido do SAO se relacione com a concentração intramuscular de substratos musculares não-dependentes do $\mathrm{O}_{2}$ (fosfocreatina - PCr) para sua utilização ${ }^{\mathbf{1 3}, \mathbf{1 4}, 15}$. Alguns estudos mostram uma diminuição monoexponencial quase imediata de $\mathrm{PCr}$ com o início da atividade de moderada a baixa intensidade ${ }^{16,17}$. Uma alternativa a esta visão de ativação rápida do SAO propõe que a resposta respiratória vem determinada por uma limitação vascular de aporte de $\mathrm{O}_{2}$ às estruturas musculares ativadas durante o exercício.

O aumento inicial dos valores de $\mathrm{VO}_{2}$, em esforços moderados, é rápido e intenso nos primeiros segundos do exercício (Figura 2), modificando sua tendência em função da intensidade empregada para essa atividade. Em nossos sujeitos, os valores de $\mathrm{VO}_{2}$ no final da primeira fase apresentaram um aumento médio de 42,21\% sobre os valores que possuíam ao início da prova, o que supõe incrementos de $8,88 \mathrm{ml} \cdot \mathrm{min}^{-1} \cdot \mathrm{W}^{-1}$. Devemos ter presente que a resposta inicial também se vê afetada pelo nível de atividade que mostre o sujeito no momento de começar a prova. Lembremos que o aquecimento é um aspecto chave na cinética inicial do $\mathrm{VO}_{2}$. Hajoglou et al. ${ }^{\mathbf{1 7}}$ comprovaram que, ao realizar esforços de elevado predomínio aeróbico ( $4 \mathrm{~min}-5 \mathrm{~min}$ ), a evolução deste parâmetro é mais rápida depois do aquecimento. Em nosso 
caso, os sujeitos iniciaram seu esforço imediatamente depois de terminar o aquecimento ( $5 \mathrm{~min}$ a $50 \mathrm{~W}$ mais $5 \mathrm{~min}$ a $100 \mathrm{~W})$.

Os valores máximos de $\mathrm{VO}_{2}$ acontecem na parte final da segunda fase como resultado da deriva que toma este parâmetro até o final do exercício. $\mathrm{O}$ valor de $\mathrm{VO}_{2}$ no momento em que se alcançam os 5т é de 90,2 $\pm 2,9 \%$ do máximo alcançado em toda a prova, o que supõe um só aumento total de 9,83\% durante a segunda parte do exercício (Tabela 5). Nesta segunda parte, os incrementos de $\mathrm{VO}_{2}$ são muito mais lentos e se mantêm em diferentes fases do exercício. Nos primeiros minutos da segunda parte do esforço, o $\mathrm{VO}_{2}$ se manteve estável para, posteriormente, ir aumentando seus valores até o final da prova. A partir desse momento, e durante alguns minutos, o aumento é progressivo e bastante estável, até alcançar valores entre 97\% e 98\% do máximo alcançado durante a prova (Tabela 6). Depois deste ponto, e até o final da prova, $\circ \mathrm{VO}_{2}$ alcançará seus valores máximos, mesmo que o habitual é achar alguma instabilidade que demonstre a aparição de certo grau de fadiga.

A intensidade de trabalho é uma variável determinante na hora de entender a cinética que segue o $\mathrm{VO}_{2}$, no esforço que realiza o organismo para contribuir com $\circ \mathrm{O}_{2}$ necessário. Isto é lógico se levarmos em conta que, para conseguir uma resposta funcional eficiente no início do exercício, é preciso iniciar, de maneira adequada, os mecanismos funcionais que permitam os necessários colaboradores de $\mathrm{O}_{2}$ para as estruturas musculares ativadas.

Diversos estudos assinalam que os fatores locais são a causa principal que atrasa a utilização do oxigênio na fase inicial do exercício $^{\mathbf{1 8 , 1 9}}$. No entanto, não existe consenso se a cinética é limitada pelo aporte de oxigênio para a zona ou por outros mecanismos locais (intramusculares) ${ }^{\mathbf{2 0 , 2 1}}$. A disponibilidade de $\mathrm{O}_{2}$ em nível local está diretamente relacionada com a quantidade de sangue que recebe a musculatura da zona de trabalho. Atualmente se desconhece, pelo menos de forma exata, o efeito que possuem as características do exercício com o fluxo do sangue das extremidades ativas (fluxo arterial) e o fluxo de sangue que existe em nível local (microcirculação). Nesse sentido, Harper et al. ${ }^{\mathbf{2 2}}$ comprovaram que durante o exercício moderado, similar ao utilizado no estudo, o fluxo do sangue no nível da artéria femoral não vai paralelo ao fluxo do sangue em nível capilar, que é o ponto onde tem lugar o intercâmbio do gás. No início da atividade, a quantidade de sangue que vai para a extremidade é significativamente superior à que circula pelos capilares da estrutura muscular ativada. Passada esta primeira fase, os valores tendem a se estabilizar e se equiparar em ambos os lugares.

A relação entre a cinética de fluxo sanguíneo arterial e o $\mathrm{VO}_{2}$ é

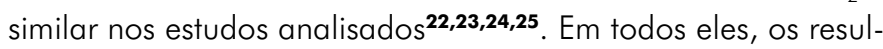
tados foram interpretados como evidência de que o aporte de $\mathrm{O}_{2}$ não limita a cinética que segue o $\mathrm{VO}_{2}$ no início do exercício. Não obstante, a diferente cinética manifestada pelos fluxos arterial e local sugere que não é apropriado utilizar o fluxo de sangue arterial para compreender a cinética da entrega de oxigênio à microcirculação. Como a cinética do sangue capilar é mais lenta do que a cinética do $\mathrm{VO}_{2}$ local, tudo parece indicar que existe uma pequena reserva de $\mathrm{O}_{2}$ capilar disponível para o músculo, mas que resulta insuficiente no início do exercício, limitando (retardando) o aumento do $\mathrm{VO}_{2}$ no início do mesmo.

Bangsbo et al. ${ }^{26}$ observaram que a extração de oxigênio pelo músculo (comprovado a partir da diferença a- $v$ de $\mathrm{O}_{2}$ na perna) se elevava poucos segundos $(<6 s$ ) antes de se iniciar o exercício. Por outro lado, em outro estudo realizado por Grassi et al. ${ }^{18}$, observou-se que as diferenças a- $v$ de $\mathrm{O}_{2}$ permaneciam inalteradas durante os primeiros 12 s de um exercício de intensidade reduzida. As diferenças achadas nestes dois estudos são explicadas por Bangsbo et al. ${ }^{\mathbf{2 6}}$ a partir do protocolo empregado para obter as amostras de sangue no nível arterial e no nível venoso. Quando a mostra é tomada ao mesmo tempo, como no estudo de Bangsbo, não se observam diferenças significativas até 13s (50\% do pico máximo) depois que iniciado o exercício, sendo necessário 51 s para alcançar $90 \%$ do pico máximo da diferença a- $v$ de $\mathrm{O}_{2}$ registrada no exercício. Os dados mostrados pelo autor sugerem que a oxigenação do músculo começa desde os primeiros segundos, situação que se produz por utilização do oxigênio armazenado no nível celular dentro da mioglobina.

Richardson et al. ${ }^{\mathbf{2 7}}$ sugerem que a metade do oxigênio armazenado na mioglobina é utilizada nos 20 s iniciais do exercício. A relação entre $\circ \mathrm{VO}_{2}$ alveolar e $\circ \mathrm{VO}_{2}$ que se produz no nível local foi estudada por DeLeroy et al. ${ }^{\mathbf{1 2}}$. Para isso, os autores estudaram em 11 sujeitos (5 homens, 6 mulheres; $23 \pm 3$ anos) a resposta ventilatória e a oxigenação local durante a aplicação de três cargas de diferentes intensidades, sempre inferiores a 80\% do limiar de lactato. Os autores assinalam que a oxigenação do músculo é mais rápida do que a cinética do $\mathrm{VO}_{2}$ expirado e, provavelmente, do que o $\mathrm{VO}_{2}$ no nível local (muscular).

Em primeira instância, a demanda de $\mathrm{O}_{2}$ depende diretamente da intensidade de trabalho e também do efeito que essa carga exerce sobre o organismo do esportista. Quando falamos de exercícios de longa duração, como os empregados no trabalho de resistência, as mudanças funcionais provocadas pela fadiga alteram o efeito agudo de cargas destas características. Nas provas podemos ver diferentes tipos de comportamento durante a segunda fase da atividade. Este comportamento individualizado depende da maior ou menor proximidade da carga ao $\mathrm{VT}_{2}$. Lembremos que, em nosso caso, os sujeitos trabalharam a uma intensidade média de $70,99 \%$ do $\mathrm{VO}_{2 \text { máx }}$ ou $87,26 \%$ do $\mathrm{VT}_{2}$.

Como conseqüência, a cinética do $\mathrm{VO}_{2}$ apresenta leves diferenças entre ambos. $\bigcirc$ sujeito da esquerda mostra o primeiro aumento (deriva ou drift do $\mathrm{VO}_{2}$ ) desde o começo da segunda fase, à qual The segue uma fase de estabilização (steady-state) e um novo aumento na parte final da prova. No segundo caso, se pode apreciar claramente a Fase 3 que, tradicionalmente, se descreve na literatura para explicar a cinética inicial do $\mathrm{VO}_{2}$ como resposta ao exercício. Esta fase estável se prolonga, neste caso, durante os 10min da segunda fase da prova, para mostrar a continuação de um aumento progressivo do $\mathrm{VO}_{2}$ até a finalização do exercício. A 
tendência média fica descrita a partir dos valores médios observados em nossos sujeitos durante a prova. Os valores mostram um aumento progressivo durante os primeiros minutos ( $\approx 12 \mathrm{~min})$, para depois se estabilizar até o final do exercício (Tabela 6 ). $\bigcirc$ aumento foi de 5,09\% $(p<0,00)$ desde o termo da primeira fase até o final do terceiro corte, com incrementos significativos entre cada um dos trechos (Tabela 7).

Posteriormente, $\mathrm{O} \mathrm{VO}_{2}$ se estabiliza durante o resto da prova sem mostrar mudanças estatisticamente significativas entre cada corte. Inclusive, no final da prova, se observa uma leve diminuição deste parâmetro durante o último trecho. É evidente que o sistema de colaboração energética sofre leves modificações ao longo dos 30min de esforço de duração de esforços de moderada intensidade. Podemos comprovar isto ao analisar o valor do coeficiente respiratório (RER) e do pulso de $\mathrm{O}_{2}\left(\mathrm{PO}_{2}\right)$. Diferente do $\mathrm{VO}_{2}$, durante a segunda fase o $\mathrm{VCO}_{2}$ sofre modificações. Como resultado, o RER mostra uma leve tendência, não estatisticamente significativa, a reduzir seus valores conforme avança a prova (Figura 3). O comportamento observado nos demonstra que, tanto o substrato energético como a via que o transforma em energia, variam com o passo do tempo por efeito do exercício. A redução de substratos, o aumento de metabólicos resultantes, o aumento da temperatura corporal e local, a resposta adaptativa multifuncional, etc., condicionam a resposta ventilatória e o sistema de aporte energético.

Lembremos que neste tipo de esforço, uma parte da energia obtida provém da gordura, fundamentalmente das triglicérides e dos ácidos gordurosos livres no sangue. Apesar das gorduras constituírem uma fonte energética que proporciona mais quilocalorias por grama do que são capazes de proporcionar os hidratos de carbono, esta fonte requer uma maior presença de oxigênio. Enquanto as gorduras produzem 5,6 moléculas de ATP por molécula de oxigênio, os hidratos de carbono permitem a produção de 6,3 moléculas. Isto faz com que este substrato aumente sua participação no abastecimento de energia, ao trabalhar de forma a manter as intensidades baixas ou moderadas (50-70\% do $\mathrm{VO}_{2 \text { máx }}$ ou menos).

Esta mudança no comportamento energético vai acompanhada de uma melhor eficiência respiratória. Podemos comprovar isto a partir da evolução que mostra o pulso de $\mathrm{O}_{2}\left(\mathrm{PO}_{2}\right)$ durante a prova. Este parâmetro de eficácia funcional foi cunhado por Ästrand e representa a quantidade de oxigênio que se consome durante a duração de um ciclo cardíaco completo (sístole mais diástole). Isto é calculado a partir dos valores médios de ambas variáveis em um tempo determinado (quase sempre 1 min). Quanto mais elevado seja seu valor, mais $\mathrm{O}_{2}$ consomem os tecidos em cada ciclo cardíaco e mais elevado resulta o custo energético. Em nosso caso, $\mathrm{PO}_{2}$ diminui com o tempo ao manter-se estável $\circ \mathrm{VO}_{2}$, apesar de aumentar sua resposta cardíaca conforme progride o exercício $\left(\mathrm{PO}_{2}\right.$ em $\mathrm{Tl}: 22,97 \pm 1,58 \mathrm{ml}$ vs. $\mathrm{PO}_{2}$ em T7: $21,37 \pm 1,61 \mathrm{ml}$.

\section{REFERÊNCIAS BIBLIOGRÁFICAS}

1. Jones AM. Oxygen uptake kinetics in sports, exercise and medicine. Londres: Routledge; 2005.

2. Hughson RL, Morrisey M. Delayed kinetics of respiratory gas exchange in the transition from prior exercise. J Appl Physiol.1982;52:921-9.

3. Gerbino A, Ward SA, Whipp, BJ. Effects of prior exercise on pulmonary gas-exchange kinetics during high-intensity exercise in humans. J Appl Physiol. 1996;80:99-107.

4. Tordi N, Perrey S, Harvey A, Hughson RL. Oxygen uptake kinetics during two bouts of heavy cycling separated by fatiguing sprint exercise in humans. J Appl Physiol. 2003;94:533-41.

5. Calderón J. Fisiología aplicada al deporte. Madrid: Editorial Tébar; 2001.

6. Willmore JH, Costill DL. Fisiología del esfuerzo y del deporte. $8^{a}$ ed. Barcelona: Editorial Paidotribo; 2007.

7. Krogh $A$, Lindhard J. The regulation of respiration and circulation during the initial stages of muscular work. J Physiol.1913;47(1-2):112-36.

8. Gamelin FX, Berthoin S, Bosquet L. Validity of the Polar S810 heart rate monitor to measure R-R intervals at rest. Med Sci Sports Exerc. 2006;38:887-93.

9. Kingsley M, Lewis MJ, Marson RE. Comparison of Polar 810 s and an ambulatory ECG system for RR interval measurement during progressive exercise. Int J Sports Med. 2005;26:39-44.

10. Rietiens GJWM, Kuipers $H$, Kester ADM, Keizer HA. Validation of a computerized metabolic measurement system (Oxycon-Pro) during low and high intensity exercise. Int J Sports Med. 2001;22:291-4.

11. Hogan MC. Fall in intracellular PO2 at the onset of contractions in Xenopus single skeletal muscle fibers. J Appl Physiol. 2001;90(5):1871-6.

12. De Lorey DS, Kowalchuk JM, Paterson DH. Relationship between pulmonary $\mathrm{O} 2$ uptake kinetics and muscle deoxygenation during moderate-intensity exercise. J Appl Physiol. 2003;95:113-20.

13. Chance B, Leigh Jr JS, Kent J, McCully K. Metabolic control principles and 31P NMR. Fed Proc. 1986;45(13):2915-20.

14. Meyer RA. A linear model of muscle respiration explains monoexponential phosphocreatine changes. Am J Physiol Cell Physiol. 1988;254:C548-53.

15. Rossiter HB, Ward SA, Doyle VL, Howe FA, Griffths JR, Whipp BJ. Inferences from pulmonary $\mathrm{O} 2$ uptake with respect to intramuscular [phosphocreatine] kinetics during moderate exercise in humans. J Physiol. 1999;518:921-32.

16. Whipp BJ, Rossiter HB, Ward SA, Avery D, Doyle VL, Howe FA, et al. Simultaneous determination of muscle $31 \mathrm{P}$ and $\mathrm{O} 2$ uptake kinetics during whole body NMR spectroscopy. J Appl Physiol. 1999;86:742-7.

17. Hajoglou A, Foster C, De Koning JJ, Lucia A, Kernozek TW, Porcari JP. Effect of Warm-Up on Cycle Time Trial Performance. Med Sci Sports Exerc. 2005;37(9):1608-14.

18. Grassi B, Poole DC, Richardson RS, Knight DR, Erickson BK, Wagner PD. Muscle O2 uptake kinetics in humans: implications for metabolic control. J Appl Physiol. 1996;80:988-98.

19. Grassi B, Gladden B, Samaja M, Stary CM, Hogan MC. Faster adjustment of O2 delivery does not affect VO2 on-kinetics in isolated in situ canine muscle. J Appl Physiol. 1998;85:1394-1403.

20. Grassi B. Regulation of oxygen consumption at the onset of exercise: is it really controversial? Exerc Sport Sci Rev. 2001;29:134-8.

21. Hughson RL, Tschakovsky ME, Houston ME. Regulation of oxygen consumption at the onset of exercise. Exerc Sport Sci Rev. 2001;29:129-33

22. Harper A, Ferreira LF, Lutjemeier BJ, Townsend DK, Barstow TJ. Human femoral artery and estimated muscle capillary blood flow kinetics following the onset of exercise. Exp Physiol. 2006;91(4):661-71.

23. Hughson RL, Shoemaker JK, Tshakovsky ME, Kowalchuk JM. Dependence of muscle VO2 on blood flow dynamics at onset of forearm exercise. J Appl Physiol. 1996;81:1619-26.

24. MacDonald MJ, Shoemaker JK, Tschakovsky ME, Hughson RL. Alveolar oxygen uptake and femoral artery blood flow dynamics in upright and supine leg exercise in humans. J Appl Physiol. 1998;85:1622-8.

25. Koga S, Poole DC, Shiojiri T, Kondo N, Fukuba Y, Miura A, et al. Comparison of oxygen uptake kinetics during knee extension and cycle exercise. Am J Physiol Regul Integr Comp Physiol. 2005;288:R212-20.

26. Bangsbo J. Muscle oxygen uptake in humans at onset of and during intense exercise. Acta Phisiol Scan. 2000;168(4):457-64.

27. Richardson RS, Noyszewski EA, Kendrick KF, Leigh JS, Wagner PD. Myoglobin O2 desaturation during exercise. Evidence of limited O2 transport. J Clin Invest. 1995;96:191226. 